\title{
Radial margin status should be determined in resected perihilar cholangiocarcinoma
}

\author{
Roeland F. de Wilde, Bas Groot Koerkamp \\ Department of Surgery, Erasmus MC University Medical Center, Rotterdam, The Netherlands \\ Correspondence to: Bas Groot Koerkamp. Department of Surgery, Erasmus MC University Medical Center, Dr. Molewaterplein 40, 3015 GD \\ Rotterdam, The Netherlands. Email: b.grootkoerkamp@erasmusmc.nl. \\ Comment on: Shinohara K, Ebata T, Shimoyama Y, et al. A Study on Radial Margin Status in Resected Perihilar Cholangiocarcinoma. Ann Surg 2019. \\ [Epub ahead of print].
}

Submitted Jul 20, 2019. Accepted for publication Jul 25, 2019.

doi: 10.21037/hbsn.2019.07.19

View this article at: http://dx.doi.org/10.21037/hbsn.2019.07.19

Curative-intent resection is the only treatment of perihilar cholangiocarcinoma (pCCA) offering patients the chance for long-term survival. Surgery generally requires an (extended) hemi-hepatectomy including the caudate lobe with resection of the extrahepatic bile duct and a regional lymph node dissection. pCCA is considered resectable when negative surgical margins can be obtained while safeguarding adequate vascular inflow to the future liver remnant. Surgical treatment of pCCA poses a challenge, because preoperative staging methods (i.e., AJCC, BismuthCorlette, and Blumgart) are inaccurate in predicting resectability. Prognosis after resection remains bleak due to the high incidence of recurrence considering 3 - and 5-year survival rates as low as $35 \%$ and $15 \%$, respectively (1). Survival rates are better in patients with a well-differentiated tumor in the absence of lymph node involvement, and after a complete resection (i.e., negative surgical margins) (2).

Positive surgical margins are frequently observed in resected pCCA, which inversely affects survival. In order to ascertain negative surgical margins, frozen section analysis is commonly performed during surgery. Herein, the proximal and distal bile duct margins are typically assessed intraoperatively. A retrospective multicenter study of pCCA patients showed that patients with an initial R1 bile duct margin with an R0 margin after additional resection had similar overall survival (OS) (HR 0.9; 95\% CI, 0.4-2.3; $\mathrm{P}=0.829$ ) as well as risk of recurrence (OR 1.5; 95\% CI, $0.8-2.7 ; \mathrm{P}=0.162)$ compared to patients with an initial $\mathrm{R} 0$ margin (3). Otsuka et al. showed that an additional R0 margin of the distal bile duct was an independent favorable prognostic factor for survival, though other studies have published contradictory results (4).

While the proximal and distal margins have frequently been assessed, the radial margin status-the circumferential tumor margin bordering the liver, hilar soft tissue and vasculature-was only assessed over a decade ago by Sakamoto et al. in distal cholangiocarcinoma and was found to be a prognostic factor in univariate analysis (5).

Only recently, the impact of positive radial margin status on OS compared to ductal margin status (i.e., proximal or distal bile duct) in resected pCCA was assessed (6). In a large single center retrospective cohort study Shinohara and colleagues described the results of 478 consecutively operated patients in a 13-year period with median follow-up of 9.4 years after application of exclusion criteria. Notably, 98 patients were excluded due to $\mathrm{pM} 1$ disease, $\mathrm{R} 2$ resection, or in-hospital death.

Results showed that $17.8 \%(85 / 478)$ of patients featured R1 margins; 37 (43.5\%) were radial margin positive, 33 (38.8\%) had positive ductal margins and in $15(17.6 \%)$ patients both margins were positive. Of the $52(37+15)$ radial margin positive cases, 32 showed margin-positivity at the hepatoduodenal ligament versus 20 at the liver resection plane. Survival analysis showed no difference between radial margin and ductal margin positivity (HR 1.14; $95 \% \mathrm{CI}$, $0.74-1.82$ ) and confirmed a significantly worse OS in case of radial margin positivity compared to margin-negative resected cases (HR 2.06; 95\% CI, 1.94-2.84). Median survival time was 4.9 years compared to 2.1 and 2.1 years in patients with R0 margin-, R1 radial margin- and R1 ductal 
margin-status, respectively. Interestingly, positive radial margin status was significantly more frequently observed in left hepatectomy (27 out of $153,17.6 \%$ ) versus other types of liver resections (24 out of 316, 7.6\%) ( $\mathrm{P}=0.001)$. Finally, the positive radial margin rate on the liver transection plane was significantly higher after left hepatectomy $(\mathrm{P}<0.001)$ and positive radial margin in the hepatoduodenal ligament was significantly more frequently observed in left-sided versus right-sided resections $(\mathrm{P}=0.031)$.

The authors should be complimented for the way in which the documented meticulous handling of the surgical specimen has been performed for nearly two decades. In doing so, they state that involvement of the surgeon in communication to surgical pathology and processing of the specimen is crucial in order to obtain reliable results. As radial margin positivity was more frequently observed in this cohort than positive ductal margins, besides improving identification of patients with worse survival, the aforementioned should be considered standard practice in centers treating pCCA for in order to optimize outcome. Without assessment of radial margin, approximately $8 \%$ (37/478) of patients would have been misclassified as R0, while they did have an R1 radial margin.

In this study there were no details on preoperative considerations with regard to the type of (intended) resection nor was there information on crossover surgical procedures or frozen section analysis. The latter is relevant to interpret the finding that there was a significantly higher incidence of $\mathrm{R} 1$ radial margin of the liver transection plane in left hepatectomy. Speculatively, this was due to a mandatory left hepatectomy in case of liver atrophy or the inability to perform a left trisectionectomy in the attempt to preserve the right anterior Glissonean pedicle. Significantly higher positive radial margins in the hepatoduodenal ligament in left hepatectomy could be explained by tumor proximity to the right hepatic artery and biliary confluence making it susceptible to tumor invasion or that simply no more margin could be obtained due to the natural boundaries of the ligament.

Put in perspective, surgical margins assessment is currently still performed by microscopy on frozen and embedded sections whereas in the future molecular margin analysis might be applicable and more accurate in determining (radial) margin status as was recently shown in pancreatic cancer (7).

In summary, surgical margin status is an independent prognostic factor for survival after resection of pCCA. Besides the commonly assessed proximal and distal ductal margins, meticulous assessment of the surgical specimen to routinely determine radial margins provides more accurate margin status in approximately one in 12 patients $(8 \%)$. Obtaining negative surgical margins remains a challenge in curative-intent resection due to the natural course of vascular and ductal structures besides the natural border of the hepatoduodenal ligament. Especially when performing left hepatectomy surgeons should be aware of tumor exposure in the hepatic hilum where the tradeoff should be considered between the risks associated with vascular reconstruction and the harm of a positive radial margin in striving for optimal outcome.

\section{Acknowledgments}

None.

\section{Footnote}

Conflicts of Interest: The authors have no conflicts of interest to declare.

Ethical Statement: The authors are accountable for all aspects of the work in ensuring that questions related to the accuracy or integrity of any part of the work are appropriately investigated and resolved.

\section{References}

1. Cillo U, Fondevila C, Donadon M, et al. Surgery for cholangiocarcinoma. Liver Int 2019;39 Suppl 1:143-55.

2. Groot Koerkamp B, Wiggers JK, Gonen M, et al. Survival after resection of perihilar cholangiocarcinomadevelopment and external validation of a prognostic nomogram. Ann Oncol 2015;26:1930-5.

3. Zhang XF, Squires MH 3rd, Bagante F, et al. The Impact of Intraoperative Re-Resection of a Positive Bile Duct Margin on Clinical Outcomes for Hilar Cholangiocarcinoma. Ann Surg Oncol 2018;25:1140-9.

4. Otsuka S, Ebata T, Yokoyama Y, et al. Clinical value of additional resection of a margin-positive distal bile duct in perihilar cholangiocarcinoma. Br J Surg 2019;106:774-82.

5. Sakamoto Y, Kosuge T, Shimada K, et al. Prognostic factors of surgical resection in middle and distal bile duct cancer: an analysis of 55 patients concerning the significance of ductal and radial margins. Surgery 2005;137:396-402.

6. Shinohara K, Ebata T, Shimoyama Y, et al. A Study 
on Radial Margin Status in Resected Perihilar

Cholangiocarcinoma. Ann Surg 2019. [Epub ahead of print].

7. Kim SJ, Kim MJ, Han JS, et al. Prediction of Recurrence
With KRAS Mutational Burden Using Ultrasensitive Digital Polymerase Chain Reaction of Radial Resection Margin of Resected Pancreatic Ductal Adenocarcinoma. Pancreas 2019;48:400-11.

Cite this article as: de Wilde RF, Groot Koerkamp B. Radial margin status should be determined in resected perihilar cholangiocarcinoma. HepatoBiliary Surg Nutr 2019;8(5):557-559. doi: 10.21037/hbsn.2019.07.19 